\begin{tabular}{|c|c|c|c|}
\hline DE & \multirow{3}{*}{$\begin{array}{l}\text { DE GRUYTER } \\
\text { OPEN }\end{array}$} & $\begin{array}{l}\text { HUNGARIAN JOURNAL OF } \\
\text { INDUSTRY AND CHEMISTRY }\end{array}$ & \multirow[t]{3}{*}{$\begin{array}{l}\text { HUUNGARIAN JOURNALI OF } \\
\text { INIDUSTIRY AND CIIIEMISTIRYY }\end{array}$} \\
\hline & & Vol. 44(2) pp. 71-79 (2016) & \\
\hline & & $\begin{array}{l}\text { hjic.mk.uni-pannon.hu } \\
\text { DOI: } 10.1515 / \text { hjic-2016-0008 }\end{array}$ & \\
\hline
\end{tabular}

\title{
ROUTE PLANNING BASED ON URBAN MOBILITY MANAGEMENT
}

\author{
TAMÁS MÁtral, ${ }^{1 *}$ JÁNOS TÓTH, ${ }^{1}$ AND MÁRTON TAMÁS HORVÁtH ${ }^{2}$ \\ ${ }^{1}$ Department of Transport Technology and Economics, Budapest University of Technology and \\ Economics, Müegyetem rkp. 3, Budapest, 1111, HUNGARY \\ 2 Department of Control for Transportation and Vehicle Systems, Budapest University of Technology \\ and Economics, Müegyetem rkp. 3, Budapest, 1111, HUNGARY
}

\begin{abstract}
Mobility management centres play a significant role in urban transport, taking into account several factors that have an effect on the flow of vehicles. In the present paper a mobility management centre equipped with necessary information and information technologies for travellers is presented that can provide route plans. A route guidance methodology is described that combines current transportation demands with the results of the traditional four-step model.
\end{abstract}

Keywords: mobility management, route planning, urban mobility, demand management

\section{Introduction}

The mobility management centre (MMC) as an organization helps to realize and manage mobility demand. The centre controls both private and public transport modes, as well as the provision of information for individual users with the aim of optimizing the entire transport network. The main goal is to provide safe and quick transportation for everyone. During the optimization process the system satisfies the mobility demands with the best available transport modes and routes, the 4R-s principles can be used (Reduce, Retime, Reroute, Revise mode) [1]. Fig.1 shows the main tasks of the mobility management centre.

Usually in an urban environment, private transport users are supported by the traffic lights from the mobility management centres. The centres can control all traffic lights in the city based on network level principles. Additionally, dynamic parking management can be developed, where the free spaces are displayed on variable message signs and in a further developed version individuals can be guided towards free spaces. Private users can be informed about the actual transport situation and weather conditions via various channels. Nowadays the most used channels are Internet-based mobile applications, but there are also radio-based solutions like RDS-TMC services or roadside infrastructure like variable message signs.

If the individual users expect a response and guidance from the MMC for their route choices and do not decide their routes individually, then these centres can optimize the demand on the level of transport network system. In this case they can manage the

*Correspondence: tamas.matrai@mail.bme.hu demand to avoid congestion - the overuse of certain routes - during peak hours, in other words, they can distribute the demand in terms of a network evenly. Currently these solutions have not yet been developed enough, due to the lack of communication among centres and users. Currently only a very small proportion of vehicles receive dynamic information from the centre. It should be mentioned here that the slow but steady shift towards shared vehicles and ondemand modes has become an emerging trend, especially in conjunction with public transport. Ideally, these services would be integrated together to provide a sustainable and equally convenient alternative to private cars for door-to-door mobility. The realisation of such

\begin{tabular}{|l|}
\hline Private Transport \\
- Traffic management (traffic \\
lights) \\
- Parking management \\
- Information provision (actual \\
traffic situation, weather) \\
- Route planning \\
- Traffic forecast \\
\hline
\end{tabular}

Public transport

- Fleet management

- Vehicle priority

- Information

provision (actual

traffic situation,

schedules,

timetables)

- Route planning
Other services

- Park and Ride information

- Bike sharing

- Car sharing

- Demand responsive transport

Figure 1. Tasks of the mobility management centre. 
an alternative has set in motion the development of a new mobility concept known as the Mobility-as-aService (MaaS), which is a user-centric, digital and intelligent mobility distribution model in which the major transport needs of users are met via a single platform and are offered by a single service provider [2]. There is a strong connection between short-term traffic forecasts and route planning. Users would like to receive route suggestions not only based on the current situation but also the forecasted one. Either they are preparing the route on their own device or they are obtaining a plan from the centre. Therefore, an efficient and acceptable level of prediction is essential.

Automatic vehicle location (AVL) is one of the key elements in the accurate running of public transport systems in urban areas. As soon as the dispatchers identify any deviation from the predefined schedule, they initiate certain actions based on a pre-defined rulebook. One action from this rulebook can be to provide priority for those vehicles that are late, this priority provision can be achieved via certain junctions which are equipped with the necessary functions. The traffic lights at these junctions can change their signal plan dynamically in order to provide a green signal to the late public transport vehicles.

Other main task of the MMC is the provision of information concerning both static timetables and current traffic situations of public transport, which can be achieved through different channels. Different information is necessary on different channels before a journey commences (e.g. Internet-based route planning services, mobile applications) and during the trip (e.g. at the public transport stop, on the vehicle). The users are expecting to get not only information, but also suggestions especially from the integrated services (e.g. connections, local public transport, railway services, coach services) based on their individual preferences [3]. The most advanced version of this approach is a dynamic multimodal journey planner which provides the ability to adjust to a variety of anomalies (i.e. network disruptions, high capacity usage, etc.) concerning the transport network and evaluates the most efficient routes to get from A to B allowing for efficient traffic management [2]. It has to be mentioned here, that a feedback loop is essential; in order to continuously fine-tune the algorithm in the background, it needs to be determined if the users are really heeding the advice of the route planner.

In functional urban areas, such as agglomerations with high levels of connectivity between urban centres and hinterland, one of the main goals is to promote the shift in mode of the commuters from private cars to more sustainable modes of transport. Therefore, the provision of information with regards to other services such as $\mathrm{P}+\mathrm{R}$ facilities and their saturation is vital. In this regard, the transfers should be seamless and the information beforehand should be sufficient to help make the right decision. There are different options to ease the level of inconvenience of the transfers, e.g. visual aids of the location of the $\mathrm{P}+\mathrm{R}$, information regarding available free spaces, guidance concerning

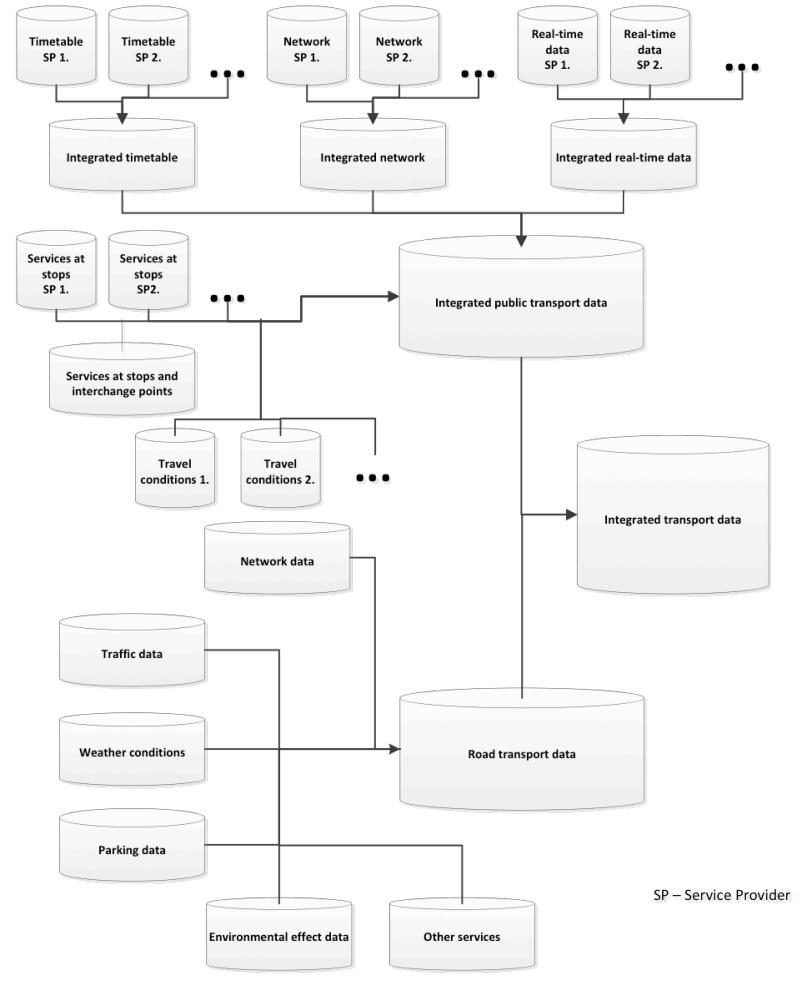

Figure 2. Schematic overview of an integrated transport database [4] (SP = service provider).

public transport services at the parking facilities, and on-the-spot information about public transport schedule.

\section{Database of Mobility Management Centres}

All the aforementioned tasks are feasible if the MMC is based on complex data collection. From these data, the MMC can create the information necessary to manage mobility demands and give information to travellers. Fig.2 shows such a possible database.

There are several public transport providers with their own data, which must be integrated into a joint database. Thus, the timetable, network, real-time data, travel conditions and services at stops or interchange points are available. Connecting timetables is particularly important when trips are satisfied by several transport modes. The intermodal hub cannot function without it. The public transport network itself is an integrated network because more public transport companies use the same routes, stops, or stations. Dynamism of data is essential for real-time services (e.g. disturbances on a network). The accurate information is sent according to the current position and status of vehicles. Vehicles equipped with a tracking system are necessary for fleet management. Tariff is an integral part of travel conditions; the passengers must be informed about prices (e.g. is there a transport association selling common tickets) and fares for different passenger groups, etc.

The data concerning fares and travel conditions remain unchanged for long periods of time; they are static or semi-dynamic data. Information systems often 


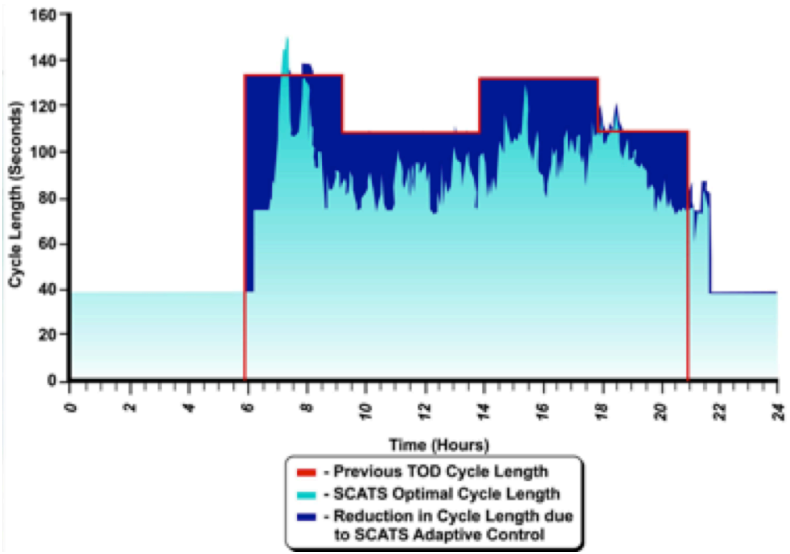

Figure 3. Changing cycle length in the SCATS system (Source: www.scats.com.au).

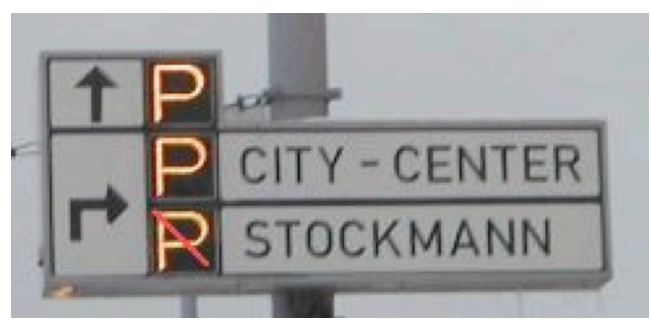

Figure 5. Variable message signs for parking as part of the HelUTC system.

lack such services at stops or stations. It could be helpful for passengers to spend time whilst waiting constructively.

The database of private transport consists of network data, traffic data (presenting the actual situation on the network and also including historical data), weather conditions, parking data, and data regarding environmental effects, and other services. The network data are static, but sometimes include semi-dynamic or dynamic data (e.g. short- or long-term road construction). Traffic data shows accidents, traffic jams, traffic volumes, etc. on the network in real-time and historically. The historical data are very important for traffic forecasting. Numerous methods are based on the comparative examination of present and past situations. Parking data can be static (location and capacity of parking area) or dynamic (parking occupation). Advanced parking systems help to find a free parking place. $\mathrm{P}+\mathrm{R}$ plays an outstanding role in encouraging drivers to change their modes transport. More and more travellers are sensitive to the environment. They are easily encouraged to use environmentally friendly modes of transport. Therefore, the importance of the data of environmental effects is more accentuated. Data regarding other services includes all the data concerning available services during trips.

\subsection{Mobility Management Centre Solutions}

According to the needs of modern society, online planning software plays an increasingly important role in optimizing the flow of traffic, and utilizing the capacity of dynamic network traffic models that are strongly connected with real-time traffic management

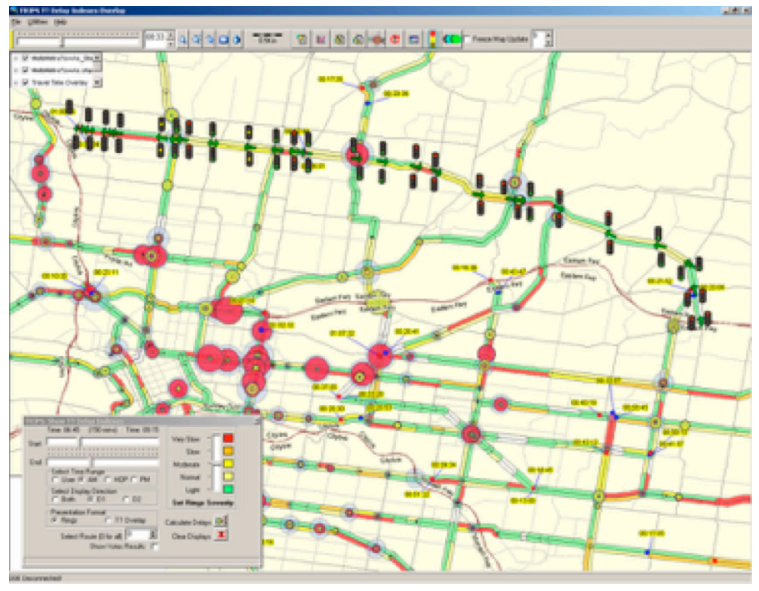

Figure 4. Estimating the expected travel time using SCATS.

systems. Several models developed for a city may not easily be applied to other cities. The rapid development of IT equipment and the efforts to standardise protocol have facilitated the development of design software for traffic management purposes. The main aim of such software is to support decision made by dispatchers based on actual traffic situations.

\subsubsection{Sydney Coordinated Adaptive Traffic System (SCATS)}

SCATS is a fully adaptive urban traffic control system that optimises traffic flow. The basic goal is to coordinate traffic lights at junctions in order to improve traffic flow and to reduce travel and waiting times. The main advantage of the system is its ability to adapt to changes in traffic conditions with the modification of the programming of traffic lights.

Fig.3 shows the daily changes of the cycle length of traffic light. The red borderline represents the previous period (before SCATS), the light blue area is the cycle length optimized by SCATS, and the dark blue area is the time saved.

Different travel demands have different goals:

- low traffic demand: minimising the number of stops;

- normal traffic demand: minimising the waiting time;

- heavy traffic: maximising the throughput capacity.

The system can prioritise public transport. In cooperation with TRIPS (traffic modelling) software, traffic forecasting is also feasible (Fig.4).

\subsubsection{Helsinki - HelUTC, Finland}

This mobility management system in Helsinki controls traffic lights, parking and public transport management systems. The concept of controlling traffic lights involves choosing a suitable cycle length according to traffic conditions. There are 10-12 different programmes for each junction. The coordinated intersections have identical cycle lengths. The system can prioritise public transport.

Variable message signs are applied to show parking information. It is very simple: are there any free spaces (Fig.5). The public transport management 


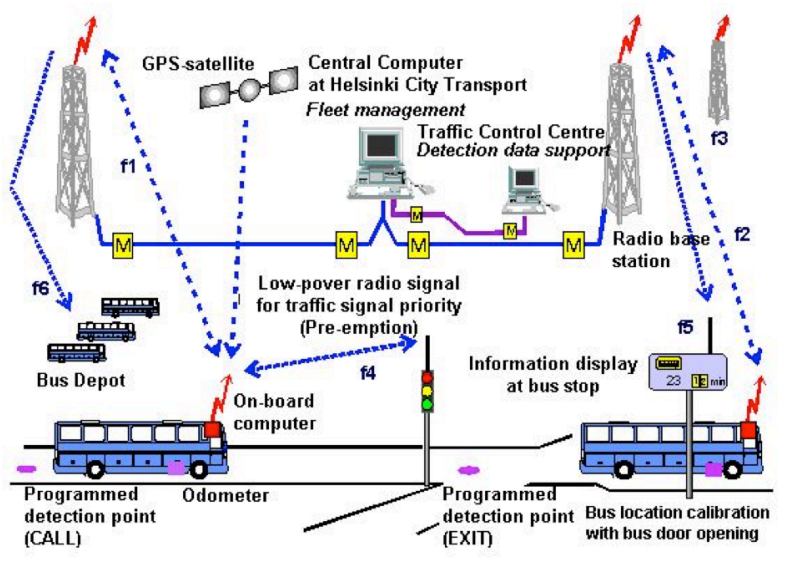

Figure 6. Diagram of the public transport management system in Helsinki.

system provides passengers with dynamic information at stops, in vehicles and pre-trips via an Internet home page or mobile application. Fig.6 shows a diagram for the system.

\subsubsection{T system in Turin, Italy}

The name of the system is "Telematic Technologies for Transport and Traffic in Torino". Fig.7 shows an overview of the system. The main goals of the system are to change the travel behaviour of passengers, increase the utilisation of public transport, and optimise travel time, energy consumption, and emissions. Subsystems of 5T consist of traffic control, public transport management including vehicle priority, environmental control, information for travellers (via Variable Message Signs, the Internet, mobile applications), and parking management.

Every subsystem has its own management centre, but on the city management level all the information required for coordinating the subsystems is concentrated. The general strategy aims to avoid the overloading of network. The centre consists of seven modules as follows:

- status monitoring module (treating subsystem information);

- origin-destination estimation module;

- modal split module (demand estimation for private and public transport);

- traffic assignment module;

- management module (cooperation with subsystems);

- operator interface module (creating a connection between the operator and system);

- database module (handling and storing the data of the operating system).

In order to decrease travel time public transport has priority at junctions, and individual drivers are informed about the status of the network and the shortest route for their trip. All the public transport vehicles equipped with AVL (Automatic Vehicle Location) to give real-time information at stops and on vehicles, via the Internet and mobile applications.

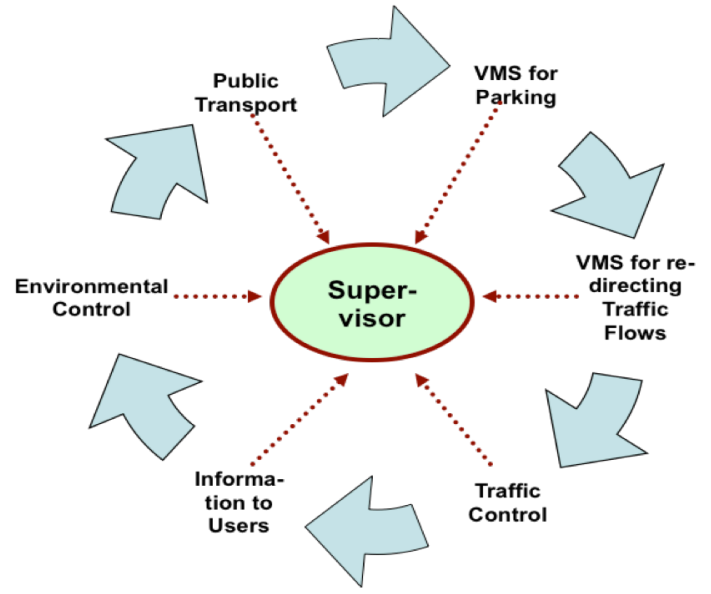

Figure 7. Overview of the 5T system.

The parking management subsystem uses VMS to show the number of free spaces. The subsystem considers historical data to account for the filling up process of the parking area. The goal is to provide information about the supposed status of the parking lot in the near future, i.e. when the vehicle arrives at the entrance.

\subsubsection{Split Cycle Offset Optimisation Technique (SCOOT)}

The SCOOT system was developed by the Transport Research Laboratory (TRL) in the UK in order to manage and control traffic lights in cities by taking into account real traffic flows. This system is used in several cities around the globe such as London, Santiago and Beijing. Since SCOOT is designed to update automatically to compensate for the effect of incidents. SCOOT achieved an average reduction of delays of about $25 \%$, when compared with up-to-date fixed-time plans in Nijmegen, The Netherlands [5].

The system also offers additional opportunities:

- providing priority to buses as part of the PT system;

- providing gate effects;

- incident detection;

- online density measurements;

- traffic flow estimation.

All static data, e.g. the physical parameters of the transport network and traffic lights are stored in the SCOOT database.

As an adaptive system, SCOOT requires good quality traffic data, so that it can respond to changes in flow. The system should calculate traffic volumes in every section of the controlled network. Detectors are normally required at every link. Their location is important and they are usually positioned at the upstream end of the approach link. Inductive loops are normally used, but other methods are also available. For the schematics of data collection and processing see Fig.8.

When vehicles pass the detector, SCOOT receives the information and converts the data into its internal units in order to construct "Cyclic flow profiles" for 


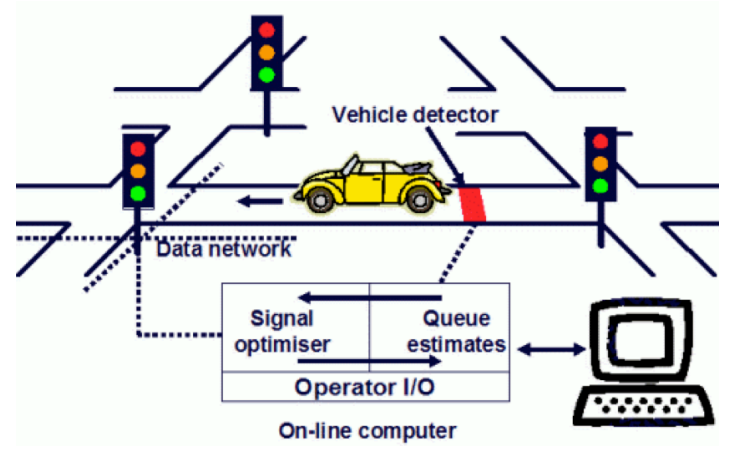

Figure 8. Data collection and analysis in SCOOT [6].

each link. The sample profile shown in the diagram is colour coded green and red according to the state of the traffic lights when vehicles arrive at the stop line at normal cruise speed. Vehicles are modelled down the link at cruise speed and join the back of the queue (if present). Whilst green, vehicles depart from the stop line at the validated saturation flow rate.

The data from the model is then used by SCOOT in three optimisers, which are continuously adapting to three key traffic control parameters - the number of green lights for each approach (Split), the time between adjacent signals (Offset) and the time allowed for all approaches to a signalised intersection (Cycle time). These three optimisers are used to continuously adapt these parameters to all intersections in the SCOOT controlled area, minimising wasted green time at intersections and reducing stops and delays by synchronising adjacent sets of signals. This means that signal timings evolve as the traffic situation changes without the inconvenience of changing fixed time plans of more traditional urban traffic control systems (see Fig.9).

The combination of these optimisers provides the necessary solution to deal with the fluctuation in different traffic scenarios, and maintain the stability of different interventions. The Split module is optimizing every status change, Offset module optimizes the duration of every phase of the signalling system, while the Cycle time module optimizes the length of the signal cycle in a certain region of the network.

Providing bus priority happens on three levels, namely (i) equal priority for all buses, (ii) different level of priority for punctual and delayed buses, and (iii) extra priority. Priority provision and intervention are independent from the detection of the buses. In order to detect PT vehicles, the cities usually develop Automatic Vehicle Location systems (AVL), in some cities Selective Vehicle Detection is applied.

Vehicle location monitoring is conducted at the AVL centre. These location data are then incorporated into the selective priority algorithm. As it can be programmed to calculate the length of delays, the vehicle with the longest delay should receive the highest priority. It is possible to provide local priorities under the supervision of SCOOT. A schematic of an example system in London can be seen in Fig.10 [6].

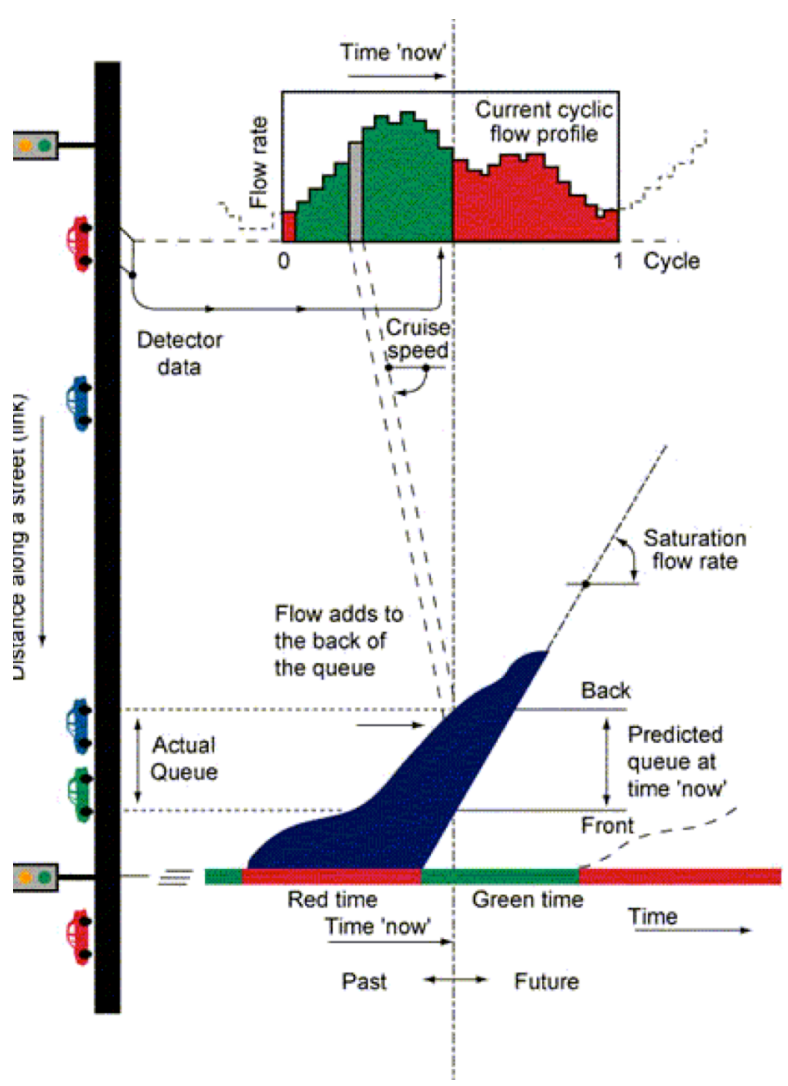

Figure 9. Illustration of the operation of the SCOOT model [6].

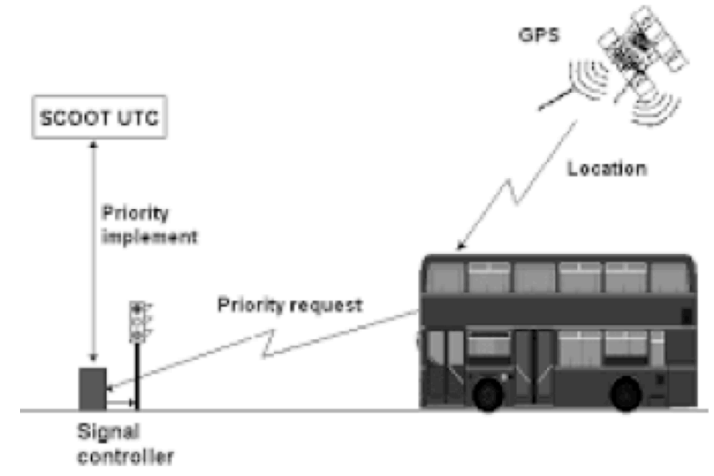

Figure 10. Schematic of the London Bus SCOOT system [6].

\subsubsection{Mobility Management in Budapest}

In Budapest the following mobility management tasks are in service:

- Public transport management and control (FUTÁR)

- Fleet management

- Vehicle priority

- Provision of information

- Route planning

- Private transport management and control

- Traffic management

- Provision of information

- Route planning

- Police monitoring centre

- Other services

○ Bike sharing 


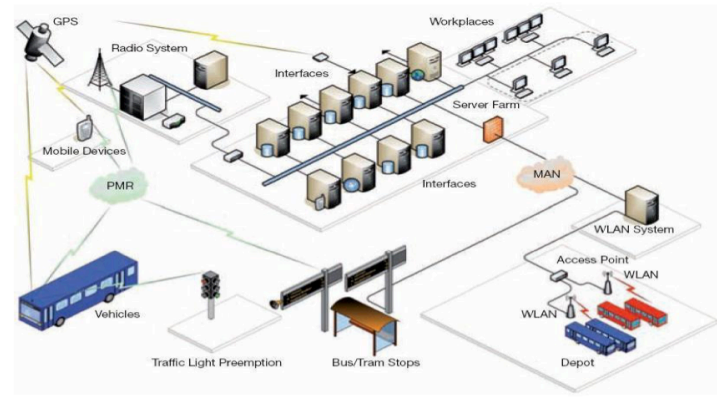

Figure 11. Illustration of the operation of the FUTÁR model.

These tasks are centralized in the same building, but there are three different management systems for the main tasks. They are connected, but there is no common supervisory system. The information flows, although not seamlessly, among them but traffic incidents are managed independently. The aim of project FUTÁR was to completely renew the provision of information and traffic management system at BKV Zrt. This renewal only concentrated on the surface transport network: trams, buses, and trolley buses. The project consisted of four main steps.

The first one was the development of the dispatcher centre and the main central server system. This consists of 32 dispatcher workplaces and all the connecting software and hardware developments. Every dispatcher can view the location of all vehicles in the network in real time and are able to intervene in order to maintain schedules especially when some incidents occur.

The second step was the developments of invehicle unit. Budapest has one of the largest surface transport vehicle fleets in Europe. Therefore, equipping these vehicles with the necessary on-board units, communication devices, and vehicle location systems was a long and demanding task. This system has been installed in 2295 vehicles and some of them have also received new displays (2024).

The third step was the deployment of roadside equipment. All over the capital 257 new displays have been deployed. These displays contain information concerning the route number of the next vehicle, while it terminate and the waiting time for this vehicle. Additionally, these displays can provide information remotely through their loudspeakers for visually impaired persons.

The last step was the introduction of the radio system. This is an integrated part of the network, which connects the dispatchers to the drivers. Moreover, the system is capable of providing priority for public transport vehicles at 30 different intersections. Fig.11 shows a schematic of the FUTÁR system.

Budapest also has a private transport management centre, which in 2012 started to use a new ITraffic version 1.6 developed by Siemens. The new system replaced the old and outdated MIGRA Central system, which was installed in 2002. One of the main reasons for the changes was that support for the operation system required by MIGRA was no longer provided. This meant that the replacement of hardware element was hardly possible. Of course, not only operating systems, hardware, or communication possibilities but also traffic management systems have evolved over the past 10 years.

The new version of the centre possesses several new features. The communication systems have been replaced with a modern IP-based system: Open Communication Interface for Road Traffic Control Systems (OCIT) and CANTO. This wireless technology has also been integrated into the system (GPRS at intersections), which is able to use any network topology or data transfer media. All the control devices which use the old BEFA 12, 15 or 16 communication systems can be supported by the new system, either as a sub-centre or as an outstation access point.

The software has also been upgraded. It has received a new clear GUI, some statistical analysis tools and further developed MOTION Logistics. Some new variable message signs have also been integrated into this system.

\section{A Pilot System in Budapest}

The aim of the pilot system presented in this paper is to demonstrate how a Mobility Management Centre equipped with the necessary information and information systems can provide tailor-made multimodal route advice to individual users by not only taking into account the average state of the transport network, but incorporating the current demand, too.

This solution can be more elaborate than already existing private services such as Waze application, for the following reasons:

- this pilot system uses O-D matrices;

- the continuous monitoring of the network is not required; therefore, the number of loop detectors, floating cars or other devices can be reduced;

- it provides real multimodal alternatives before trips in order to choose the best transport mode for the current trip;

- it uses already existing services; therefore, it does not require any major investment;

- it is a module-based system; therefore, easily configurable to match local needs.

The distribution of the demand on the network cannot be estimated without a transport model, which assigns the demand to the available transport network. Fig.12 shows the proposed concept of this pilot system.

The main concept behind this approach is to use the conventional, static demand matrices for the first iteration of the network load, but later on these matrices will be fine-tuned based on current demands. During the route planning phase, actual road traffic disturbances, e.g. road construction, accident; dynamic information from the AVL system; and the current availability of other services like bikes at the nearest bike sharing station, are taken into account. Based on the combination of this information and predefined user preferences, the system provides a tailor-made route for the user that is compared with at least two alternatives. 
The system will use time intervals of 15 minutes to calculate the average situation, based on pre-defined static demand matrices (SDM) for each 15 minute-long time interval of the day, starting from midnight. The current demand, which is the sum of the number of queries concerning a specific route between distinct points of the network, is recorded in an actual demand matrix (ADM). In order to obtain the current state of the network the sum of the SDM and ADM is necessary. However, SDM already indirectly contains the demands of the ADM. However, only as a static estimation from which the real state, represented by the ADM, can differ. Therefore, the ADM cannot be added directly to the SDM, so the system will use a complex method to create a dynamic demand matrix (DDM), which correctly incorporates the actual demand. The static demand matrix is reduced proportionally by the corner sum of the actual demand matrix. Then the ADM and SDM are added together resulting in the new matrix (DDM) for the next 15 minute period. Route suggestions will be provided based on the current state of the transport network.

\section{Discussion}

As described in the Methodology chapter, the concept of the pilot project has already been elaborated. The existing matrix of the Budapest Macroscopic Transport Model [5] will be used as a daily static matrix and create matrices for each 15 minute interval of the day. Demand matrices will be created for every mode individually (4 freight categories, car, bike, public transport). However, only car and bike matrices will be updated with the current demand.

The averaging method described in the previous chapter is valid until the sum of the actual demand matrix corner sum reaches $30 \%$ of the static demand. Over this scale, the averaging might yield false results and a different method will need to be applied. An important assumption is that the sum of the corner of the average daily matrix is fixed, in other words, each day the same number of trips is conducted on the network.

A public transport model will not be developed in this pilot case, since an online journey planner exists which is based on real-time data and can make queries. Alternatively, the existing interface of the FUTÁR system will be used to provide public transport route suggestions. For car and bike users, a search for the shortest route will be based on the results of traffic assignments obtained by VISUM software. Disturbance to the road network will be added as changes in link or node capacity occur. In this case, a simple approach can be formulated, but in the end it represents the real disruption to the network.

A description of the entire procedure is shown in Fig.13. The entire procedure consists of two main phases: the preparation and elaboration periods. The preparation period can be done any time before the system starts. The elaboration period can be divided into 15 minute intervals that are represented in the figure by

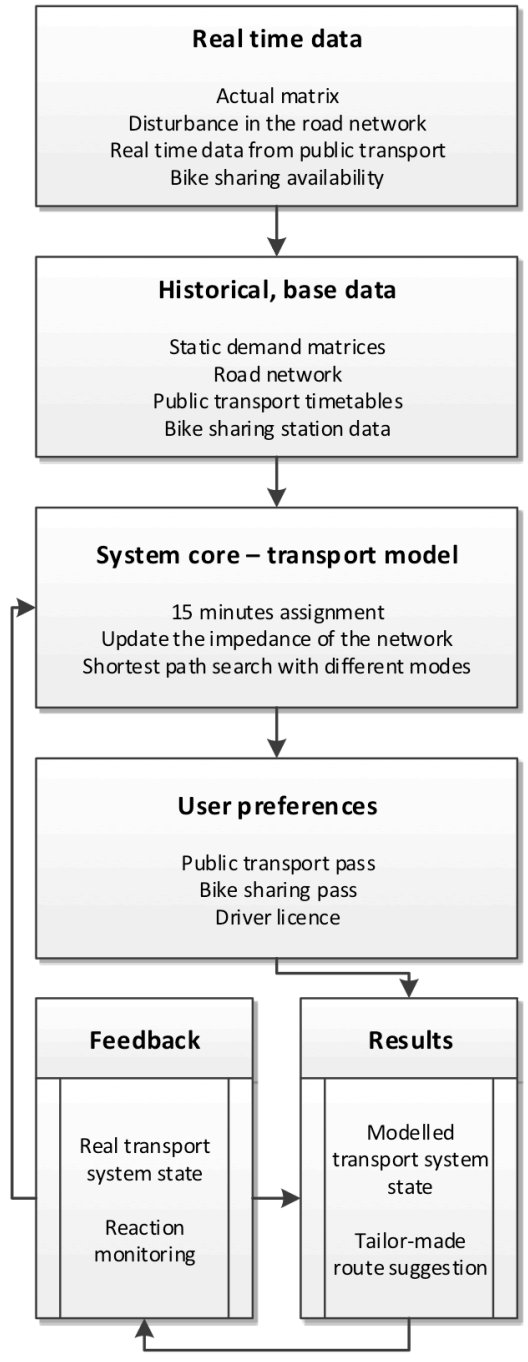

Figure 12. Concept overview of the pilot system introduced in Budapest.

Time Periods of between 1 and T. Time Period 0 is an initialising period that differs slightly from the others, since there are no measurements beforehand. The indexing of time periods and time are the following: $\tau$ represents the time when a routing request arrives in the system, $(t-1)$ and $(t)$ are the start and end times of $T, T$ is the time period when $\tau$ occurs. During the preparation period the 15 minute-long matrices are created for each mode and the basic static equilibrium assignment STA[PREP] has to be run. These assignments run for roughly 45 minutes for each Time Period on an average PC using VISUM. The results of these assignments are the basic loads of the network.

Time Period 0 is the initial period, there is no routing request that is filled before that period. Therefore, disruptions only exist for the average state. The routing can be drawn from this state and the requests are collected in an actual demand matrix. In this period a traffic assignment STA[0] runs which incorporates the traffic disturbance reported at the beginning (period -1) of the period and the static demand matrix for the next period (period 1). This assignment usually runs for 8-10 minutes on an average PC running VISUM. 

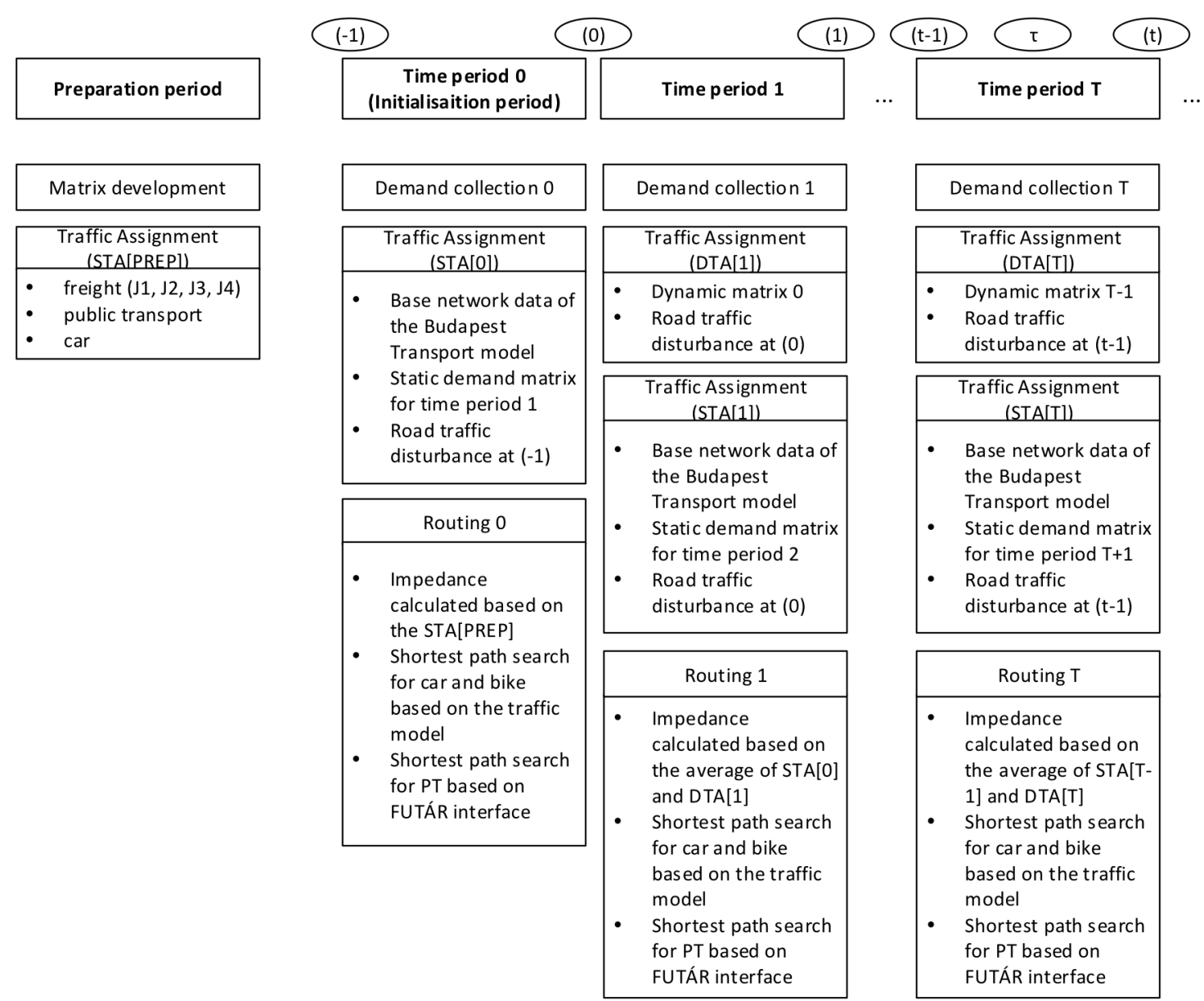

disturbance at (t-1)
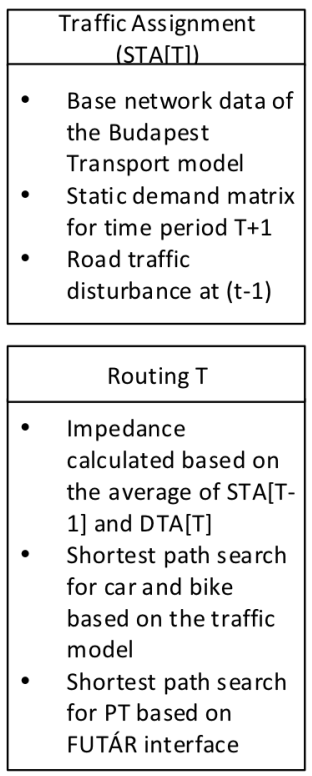

Figure 13. List of processes of the pilot system introduced in Budapest.

Time Period T is the current period, when current routing requests arrive and are collected in an actual demand matrix. An All-or-Nothing assignment DTA[T] is run using the dynamic matrix $\mathrm{T}-1$ and the corrected capacity based on road traffic disturbances in (t-1). This assignment usually runs for less than 5 seconds. STA[T] is the same type of assignment as STA[0], but with the relevant data. The routing algorithm uses an average level of disturbance from STA[T-1] and DTA[T] to determine the shortest route for car and bike users. Time period $T$ can represent any subsequent period as well, where the procedure is identical.

\section{Conclusion}

This paper introduces the concept of mobility management centres. It provides an overview of the tasks, responsibilities and opportunities of mobility management centres together with some international best practices. Later an innovative pilot system is described in detail that will be elaborated in Budapest.

Certainly, as this is a pilot system, it has several limitations and points for further research have been identified. Some of the limitations arise from the required run times of the transport model, e.g. a shorter time interval than 15 minutes cannot be used. From the description of the process, it is evident that it includes averaging in several instances.
This system assumes that all the planned journeys will be made; however, under real circumstances there are more planned than actual journeys. This can be one area of further research, since a good approach can be used to determine the proportion of planned journeys that are actually made. As suggested, a model like this should provide feedback, but this will not be developed during the pilot study.

The validation of the proposed system is important. It can be validated by data from inductive loops and floating cars, but the above mentioned feedback loop would be an asset. There is another validation step, which should be done regarding the capacity reduction of the road traffic disturbance. The current system operates with some educated guesses, but appropriate values can be calculated with actual data. The planned approach for this is to collect incident data from the road network and at the same time collect data from loop detectors in close proximity to the incidents.

The current pilot system is based on a series of static equilibrium assignments, but such a system should use Dynamic User Equilibria or Dynamic Stochastic Assignments. The authors are currently analysing the possibilities of this approach, but it might require greater computing demands as well as more input data, which are currently not available in Budapest. Nevertheless, this is one of the most important research steps. 


\section{Acknowledgement}

We acknowledge the financial support of this work by the Hungarian State under the VKSZ_12-1-2013-0088 project. The authors are grateful for the comments from colleagues at the BME Department of Transport Technology and Economics.

\section{REFERENCES}

[1] Juhász, M.; Mátrai, T.; Gál, G.; Kerényi, L.S.: Mobility management survey for the 2012 Olympic games on the basis of published data for "Transport for London", Közlekedéstudományi Szemle 20132 , 11-19 (in Hungarian)

[2] Kamargianni, M.; Matyas, M.; Li, W.; Schäfer, A.: Feasibility study for 'Mobility as a Service' concept in London, UCL Energy Institute, Dept. Transp. 2015, 1-82 DOI: 10.13140/RG.2.1.3808.1124
[3] Esztergár-Kiss, D.; Csiszár, C.: Evaluation of multimodal journey planners and definition of service levels, Int. J. Intel. Transp. Syst. Res. 2015 13(3), 154-165 DOI: 10.1007/s13177-014-0093-0

[4] Tóth, J.: ITS international trends, Proc. XI. Current Issues of Urban Transport: Organisations, Innovation, Quality Service (Közlekedéstudományi Egyesület, Balatonfenyves, Hungary) 2011 (in Hungarian)

[5] Mátrai, T.; Ábel, M.; Kerényi, L.S.: How can a transport model be integrated to the strategic transport planning approach: A case study from Budapest, Proc. 2015 Int. Conf. Model. Technol. Intel. Transp. Syst. pp. 192-199, 2015 DOI: 10.1109/MTITS.2015.7223256

[6] SCOOT Advice Leaflet 1: The "SCOOT" urban traffic control system, available at scootutc.com/documents/1_SCOOT-UTC.pdf. 